\title{
Conservation Properties of Symmetric BVMs Applied to Linear Hamiltonian Problems *
}

\author{
Pierluigi Amodio ${ }^{1}$, Felice Iavernaro ${ }^{1}$, and Donato Trigiante ${ }^{2}$ \\ 1 Dipartimento di Matematica, Università di Bari, \\ Via E. Orabona 4, I-70125 Bari, Italy, \\ amodio@dm.uniba.it, felix@dm.uniba.it \\ 2 Dipartimento di Energetica, Università di Firenze, \\ Via C. Lombroso 6/17, I-50134 Firenze, Italy, \\ trigiant@cesit1.unifi.it
}

\begin{abstract}
We consider the application of symmetric Boundary Value Methods to linear autonomous Hamiltonian systems. The numerical approximation of the Hamiltonian function exhibits a superconvergence property, namely its order of convergence is $p+2$ for a $p$ order symmetric method. We exploit this result to define a natural projection procedure that slightly modifies the numerical solution so that, without changing the convergence properties of the numerical method, it provides orbits lying on the same quadratic manifold as the continuous ones. A numerical test is also reported.
\end{abstract}

\section{Introduction}

Hamiltonian systems are not structurally stable against non-Hamiltonian perturbations, like those introduced by an ordinary numerical method during the discretization procedure. As a consequence, loss of some peculiar properties, such as the conservation of invariants or simplecticity, may be checked in the numerical solution, unless suitable classes of numerical integrators are used. This problem has led to the introduction of a number of methods and techniques to preserve the features of the Hamiltonian structure (see for example [5]; recent results on the subject may be found in [4] and references therein). We consider the linear Hamiltonian problem

$$
\mathbf{y}^{\prime}=L \mathbf{y}, \quad t \in\left[t_{0}, t_{0}+T\right]
$$

where $L$ is a Hamiltonian matrix of the form $L=J S, S$ is a square real symmetric matrix of dimension $2 m$ and

$$
J=\left(\begin{array}{rr}
0 & -I \\
I & 0
\end{array}\right)
$$

( $I$ is the identity matrix of dimension $m$ ). We solve this problem numerically using a symmetric Boundary Value Method (BVM). The existence of a symplectic

\footnotetext{
* Work supported by GNCS.
} 
generating matrix associated to a symmetric BVM has been shown in [3]. The main result of the present paper is a superconvergence property those schemes are proved to share. This will allow us to implement a trivial projection procedure that provides orbits lying on the same quadratic manifold as the continuous ones and preserving the order of the underlying method.

Introducing a uniform mesh $t_{0}, t_{1}, \ldots, t_{N}$ over the integration interval $\left[t_{0}, t_{0}+\right.$ $T$ ] with stepsize $h=T / N$, a symmetric BVM applied to (1) is defined by the linear multistep formula

$$
\sum_{j=0}^{r} \alpha_{j}\left(\mathbf{y}_{n+j}-\mathbf{y}_{n-j-1}\right)-h \sum_{j=0}^{r} \beta_{j} L\left(\mathbf{y}_{n+j}+\mathbf{y}_{n-j-1}\right)=\mathbf{0}
$$

that must be solved for $n=r+1, r+2, \ldots, N-r$. The additional conditions

$$
\mathbf{y}_{0}, \ldots, \mathbf{y}_{r} \quad \text { and } \quad \mathbf{y}_{N-r+1}, \ldots, \mathbf{y}_{N}
$$

are obtained adding the initial condition $\mathbf{y}\left(t_{0}\right)=\mathbf{y}_{0}$ and $2 r$ extra multistep formulae called initial and final methods. The coefficients $\alpha_{j}$ and $\beta_{j}$ are determined imposing that $\mathbf{y}_{n}$ is an approximation of order $p$ of the continuous solution $\mathbf{y}\left(t_{n}\right)$; we will also assume $\sum_{j=-r_{1}}^{r_{2}} \beta_{j}=1$ as a normalization condition to avoid the indetermination of the coefficients $\alpha_{j}$ and $\beta_{j}$. In matrix notation a symmetric BVM takes the form

$$
(A \otimes I-h B \otimes L) Y=-\mathbf{a} \otimes \mathbf{y}_{0}+h \mathbf{b} \otimes\left(L \mathbf{y}_{0}\right) .
$$

where $Y=\left[\mathbf{y}_{0}^{T}, \mathbf{y}_{1}^{T}, \ldots, \mathbf{y}_{N}^{T}\right]^{T}$ is the solution vector, $A$ and $B$ are square matrices of dimension $N$, and the right hand side contains a known term that accounts for the initial condition. Apart from the initial and final blocks, each consisting of $r$ rows containing the coefficients of the additional initial and final methods respectively, the matrices $A$ and $B$ have a Toeplitz structure defined by the $r+1$ coefficients of the main method; here is, for instance, how $A$ looks like:

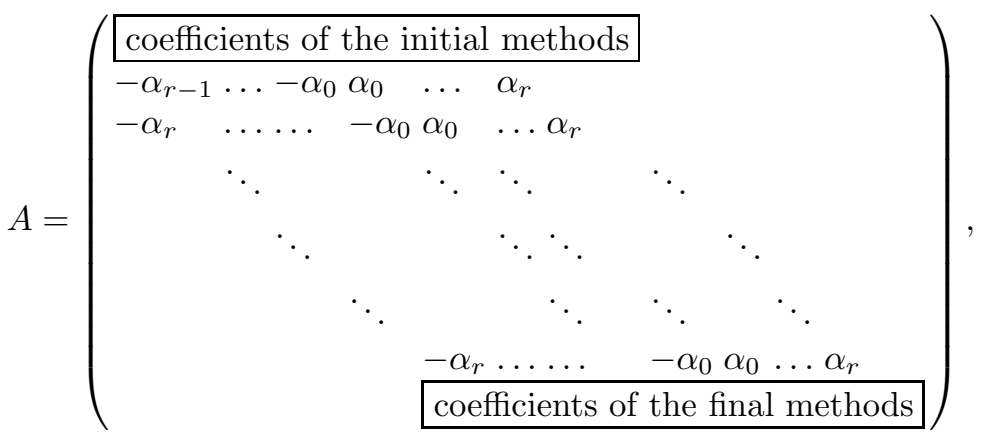

and analogously for $B$. The vectors $\mathbf{a}$ and $\mathbf{b}$ contain the coefficients of the formulae in (3) that are combined with the initial condition $\mathbf{y}_{0}$; sometimes it is useful to insert such vectors as extra-columns in the matrices $A$ and $B$. This is accomplished by defining the two extended matrices $\widetilde{A}=[\mathbf{a}, A], \widetilde{B}=[\mathbf{b}, B]$, of 
size $N \times(N+1)$ and the vector $\tilde{Y}=\left[\mathbf{y}_{0}^{T}, Y^{T}\right]^{T}$; in terms of these quantities, the system (3) becomes

$$
(\widetilde{A} \otimes I-h \widetilde{B} \otimes L) \widetilde{Y}=\mathbf{0} .
$$

The Extended Trapezoidal Rules of the first and second kind (ETRs and ETR $_{2} \mathrm{~s}$ ) and the Top Order Methods (TOMs) are three examples of classes of symmetric schemes (see [3] for their definition and properties). ETRs are defined as

$$
\mathbf{y}_{n}-\mathbf{y}_{n-1}-h \sum_{j=0}^{r} \beta_{j} L\left(\mathbf{y}_{n+j}+\mathbf{y}_{n-j-1}\right)=\mathbf{0},
$$

and have order $p=2 r+2$. The formula of order 4 is used in the last section in a numerical test.

\section{Superconvergence of the numerical Hamiltonian function}

We denote by $\sigma=\left(\mathbf{y}\left(t_{n}\right)\right)^{T} S \mathbf{y}\left(t_{n}\right)$ the value (independent of $n$ ) of the Hamiltonian function over the continuous solution and by $\sigma_{n}=\mathbf{y}_{n}^{T} S \mathbf{y}_{n}$ the approximation of such value, generated by the numerical method.

Since $\mathbf{y}_{n}=\mathbf{y}\left(t_{n}\right)+O\left(h^{p}\right)$, it follows that

$$
\sigma_{n}=\sigma+O\left(h^{p}\right)
$$

The rate of convergence of the Hamiltonian function evaluated over the numerical solution, towards its limit value $\sigma$, is therefore (in general) inherited by the order of the underlying method. Hereafter, we prove that symmetric BVMs of even order $p$ provide a two orders higher rate of convergence, that is $\sigma_{n}=\sigma+O\left(h^{p+2}\right)$.

Given a vector $\mathbf{w}$, we will denote by $\mathbf{w}^{k}$ the vector whose $n$th component is $\left(w_{n}\right)^{k}$. Furthermore $\tilde{\mathbf{e}}$ and $\mathbf{e}$ will be two vectors with components equal to one and of length $N+1$ and $N$ respectively, while $\mathbf{u}=[1,2, \ldots, N]^{T}$ and $\tilde{\mathbf{u}}=\left[0, \mathbf{u}^{T}\right]^{T}$. The order conditions on a BVM may be recasted in block form:

$$
\begin{array}{ll}
\widetilde{A} \tilde{\mathbf{e}}=\mathbf{0}, \quad \widetilde{A} \tilde{\mathbf{u}}-\widetilde{B} \tilde{\mathbf{e}}=\mathbf{0} & \text { (consistency conditions), } \\
\widetilde{A} \tilde{\mathbf{u}}^{k}-k \widetilde{B} \tilde{\mathbf{u}}^{k-1}=\mathbf{0}, & k=2, \ldots, p .
\end{array}
$$

To begin with, we list some properties of the BVMs that will be exploited in the sequel. The proof of Lemma 1 is trivial and will be omitted.

Lemma 1. For any BVM (3) the following relations hold true:
(a) $\widetilde{B} \tilde{\mathbf{e}}=\mathbf{e}$;
(b) $A^{-1} \mathbf{a}=-\mathbf{e}$;
(c) $A^{-1} \mathbf{b}=\mathbf{u}-A^{-1} B \mathbf{e}$;
(d) $A^{-1} \mathbf{e}=\mathbf{u}$.

Lemma 2. A BVM of order $p$ satisfies the following $p-1$ conditions:

$$
\left(A^{-1} B\right)^{k-1} \mathbf{u}=\frac{1}{k !} \mathbf{u}^{k}, \quad k=2, \ldots, p .
$$


Proof. For $k=2, \ldots, p$ the order conditions (7) are simplified as follows

$$
A \mathbf{u}^{k}-k B \mathbf{u}^{k-1}=\mathbf{0},
$$

the first element of $\tilde{\mathbf{u}}$ being zero. Multiplying on the left by $A^{-1}$ yields

$$
\mathbf{u}^{k}=k A^{-1} B \mathbf{u}^{k-1}=k(k-1)\left(A^{-1} B\right)^{2} \mathbf{u}^{k-2}=\ldots=k !\left(A^{-1} B\right)^{k-1} \mathbf{u}
$$

and (8) follows.

Lemma 3. Given a symmetric BVM with $2 r+1$ steps and a vector $\boldsymbol{\xi}$ of length $N$ and all null entries except (possibly) the first and the last $r$, one has:
(a) $A^{-1} \boldsymbol{\xi}=g \mathbf{e}+\hat{\boldsymbol{\xi}}$,
(b) $A^{-1} \hat{\boldsymbol{\xi}}=g_{1} \mathbf{e}+\hat{\boldsymbol{\xi}}_{1}$,
(c) $B \mathbf{u}=\mathbf{u}-\frac{1}{2} \mathbf{e}+\boldsymbol{\xi}$,
(d) $A^{-1} \mathbf{u}=\frac{1}{2}\left(\mathbf{u}^{2}+\mathbf{u}\right)-g \mathbf{e}-\hat{\boldsymbol{\xi}}$,

where $g$ and $g_{1}$ are constant and $\hat{\boldsymbol{\xi}}$ and $\hat{\boldsymbol{\xi}}_{1}$ are vectors whose components decrease in modulus with an exponential rate when moving from the edge towards the inside of the vector (in the sequel the vectors denoted by $\boldsymbol{\xi}$ and $\hat{\boldsymbol{\xi}}$ are assumed to share the same kind of shape as described above).

Proof. The Toeplitz matrix associated to $A$ has $r+1$ lower and $r$ upper offdiagonals and is generated by the first characteristic polynomial of the basic method. This polynomial has $r$ roots inside, $r$ outside and one on the boundary of the unit circle (see [3]). It follows that, starting from the main diagonal, the entries in each column of the matrix $A^{-1}$ tend to a constant value when we move downwards, and decrease exponentially in modulus when we move upwards [2]. From this consideration and the definitions of $\boldsymbol{\xi}$ and $\hat{\boldsymbol{\xi}}$, (a) and (b) immediately follow.

The $n$-th component of the vector $B \mathbf{u}$ is

$$
(B \mathbf{u})_{n}=\sum_{j=0}^{r} \beta_{j}[(n+j)+(n-j-1)]=(2 n-1) \sum_{j=0}^{r} \beta_{j}=n-\frac{1}{2},
$$

which gives (c) (we notice that the non null elements of $\boldsymbol{\xi}$ depend on the initial and final methods). Since a consistent symmetric method has order at least two, we have that $A \mathbf{u}^{2}-2 B \mathbf{u}=\mathbf{0}$ and exploiting in sequence (c), (d) of Lemma 1 and (a), we obtain (d).

Since our goal is the computation of $\sigma_{n}$, we first derive an expression for $\mathbf{y}_{n}$ in terms of $\mathbf{y}_{0}$. From (3) we obtain (for small $h$ ):

$$
\begin{aligned}
Y & =(A \otimes I-h B \otimes L)^{-1}\left(-\mathbf{a} \otimes \mathbf{y}_{0}+h \mathbf{b} \otimes\left(L \mathbf{y}_{0}\right)\right) \\
& =\left(I_{N} \otimes I-h A^{-1} B \otimes L\right)^{-1}\left(-A^{-1} \mathbf{a} \otimes \mathbf{y}_{0}+h A^{-1} \mathbf{b} \otimes\left(L \mathbf{y}_{0}\right)\right) \\
& =\left(\sum_{j=0}^{\infty} h^{j}\left(A^{-1} B\right)^{j} \otimes L^{j}\right)\left(\mathbf{e} \otimes \mathbf{y}_{0}+h A^{-1} \mathbf{b} \otimes\left(L \mathbf{y}_{0}\right)\right)
\end{aligned}
$$




$$
\begin{aligned}
& =\sum_{j=0}^{\infty} h^{j}\left(A^{-1} B\right)^{j} \mathbf{e} \otimes L^{j} \mathbf{y}_{0}+\sum_{j=0}^{\infty} h^{j+1}\left(A^{-1} B\right)^{j} A^{-1} \mathbf{b} \otimes L^{j+1} \mathbf{y}_{0} \\
& =\mathbf{e} \otimes \mathbf{y}_{0}+\sum_{j=1}^{\infty} h^{j}\left[\left(A^{-1} B\right)^{j} \mathbf{e}+\left(A^{-1} B\right)^{j-1} \mathbf{u}-\left(A^{-1} B\right)^{j} \mathbf{e}\right] \otimes L^{j} \mathbf{y}_{0} \\
& =\mathbf{e} \otimes \mathbf{y}_{0}+\sum_{j=1}^{\infty} h^{j}\left(A^{-1} B\right)^{j-1} \mathbf{u} \otimes L^{j} \mathbf{y}_{0}
\end{aligned}
$$

(property (b) and (c) of Lemma 1 has been exploited to derive the third and fifth equalities). Denoting by $\mathbf{e}_{n}$ the $n$-th vector of the canonical basis on $\mathbb{R}^{N}$, we obtain

$$
\begin{aligned}
\mathbf{y}_{n} & =\left(\mathbf{e}_{n}^{T} \otimes I\right) Y=\mathbf{y}_{0}+\sum_{j=1}^{\infty} h^{j}\left(\mathbf{e}_{n}^{T} \otimes I\right)\left[\left(A^{-1} B\right)^{j-1} \mathbf{u} \otimes L^{j} \mathbf{y}_{0}\right] \\
& =\mathbf{y}_{0}+\sum_{j=1}^{\infty} h^{j}\left(\mathbf{e}_{n}^{T}\left(A^{-1} B\right)^{j-1} \mathbf{u}\right) L^{j} \mathbf{y}_{0} .
\end{aligned}
$$

For the computation of $\sigma_{n}$ we will make use of the relation

$$
\left(\sum_{j=1}^{\infty} \mathbf{v}_{j}\right)^{T}\left(\sum_{j=1}^{\infty} \mathbf{w}_{j}\right)=\sum_{j=1}^{\infty} \sum_{k=1}^{j} \mathbf{v}_{k}^{T} \mathbf{w}_{j-k+1}
$$

where $\left\{\mathbf{v}_{j}\right\}$ and $\left\{\mathbf{w}_{j}\right\}$ are two sequences of vectors whose related series are supposed to converge. We have

$$
\begin{aligned}
\sigma_{n} & =\mathbf{y}_{0}^{T} S \mathbf{y}_{0}+2 \mathbf{y}_{0}^{T} S\left(\sum_{j=1}^{\infty} h^{j}\left(\mathbf{e}_{n}^{T}\left(A^{-1} B\right)^{j-1} \mathbf{u}\right) L^{j} \mathbf{y}_{0}\right) \\
+ & \sum_{j=1}^{\infty} \sum_{k=1}^{j}\left[h^{k}\left(\mathbf{e}_{n}^{T}\left(A^{-1} B\right)^{k-1} \mathbf{u}\right) \mathbf{y}_{0}^{T}\left(L^{T}\right)^{k} S h^{j-k+1}\left(\mathbf{e}_{n}^{T}\left(A^{-1} B\right)^{j-k} \mathbf{u}\right) L^{j-k+1} \mathbf{y}_{0}\right] \\
& =\sigma+2 \sum_{j=1}^{\infty} h^{j}\left(\mathbf{e}_{n}^{T}\left(A^{-1} B\right)^{j-1} \mathbf{u}\right)\left(\mathbf{y}_{0}^{T} S(J S)^{j} \mathbf{y}_{0}\right) \\
& +\sum_{j=1}^{\infty} h^{j+1} \sum_{k=1}^{j}\left[\left(\mathbf{e}_{n}^{T}\left(A^{-1} B\right)^{k-1} \mathbf{u}\right)\left(\mathbf{e}_{n}^{T}\left(A^{-1} B\right)^{j-k} \mathbf{u}\right)\left(\mathbf{y}_{0}^{T}\left(L^{T}\right)^{k} S L^{j-k+1} \mathbf{y}_{0}\right)\right] \\
& =\sigma+2 \sum_{j=1}^{\infty} h^{j}\left(\mathbf{y}_{0}^{T} S(J S)^{j} \mathbf{y}_{0}\right)\left(\mathbf{e}_{n}^{T}\left(A^{-1} B\right)^{j-1} \mathbf{u}\right) \\
& +\sum_{j=1}^{\infty} h^{j+1}\left(\mathbf{y}_{0}^{T} S(J S)^{j+1} \mathbf{y}_{0}\right) \sum_{k=1}^{j}(-1)^{k}\left(\mathbf{e}_{n}^{T}\left(A^{-1} B\right)^{k-1} \mathbf{u}\right)\left(\mathbf{e}_{n}^{T}\left(A^{-1} B\right)^{j-k} \mathbf{u}\right)
\end{aligned}
$$




$$
\begin{aligned}
& =\sigma+2 h\left(\mathbf{y}_{0}^{T} S J S \mathbf{y}_{0}\right)\left(\mathbf{e}_{n}^{T} \mathbf{u}\right)+2 \sum_{j=2}^{\infty} h^{j}\left(\mathbf{y}_{0}^{T} S(J S)^{j} \mathbf{y}_{0}\right)\left(\mathbf{e}_{n}^{T}\left(A^{-1} B\right)^{j-1} \mathbf{u}\right) \\
& +\sum_{j=2}^{\infty} h^{j}\left(\mathbf{y}_{0}^{T} S(J S)^{j} \mathbf{y}_{0}\right) \sum_{k=1}^{j-1}(-1)^{k}\left(\mathbf{e}_{n}^{T}\left(A^{-1} B\right)^{k-1} \mathbf{u}\right)\left(\mathbf{e}_{n}^{T}\left(A^{-1} B\right)^{j-k-1} \mathbf{u}\right) .
\end{aligned}
$$

For any vector $\mathbf{z} \in \mathbb{R}^{2 m}$ one has $\mathbf{z}^{T} J \mathbf{z}=0$, which gives $\mathbf{z}^{T} S(J S)^{j} \mathbf{z}=0$ for any positive and odd integer $j$. A consequence is that the second term in the above expression of $\sigma_{n}$ vanishes and both series will contain only terms with even powers in $h$ :

$$
\begin{aligned}
\sigma_{n}= & \sigma+\sum_{j=1}^{\infty} h^{2 j}\left(\mathbf{y}_{0}^{T} S(J S)^{2 j} \mathbf{y}_{0}\right)\left[2\left(\mathbf{e}_{n}^{T}\left(A^{-1} B\right)^{2 j-1} \mathbf{u}\right)\right. \\
& \left.+\sum_{k=1}^{2 j-1}(-1)^{k}\left(\mathbf{e}_{n}^{T}\left(A^{-1} B\right)^{k-1} \mathbf{u}\right)\left(\mathbf{e}_{n}^{T}\left(A^{-1} B\right)^{2 j-k-1} \mathbf{u}\right)\right] .
\end{aligned}
$$

From (6) we realize that the series cannot contain terms of order lower than $h^{p}$. Indeed we show that the first $p / 2$ terms of the series vanish. For $j=1, \ldots, p / 2$, considering the relations (8) (that can be extended to $p=1$ ) we have

$$
\begin{aligned}
& 2\left(\mathbf{e}_{n}^{T}\left(A^{-1} B\right)^{2 j-1} \mathbf{u}\right)+\sum_{k=1}^{2 j-1}(-1)^{k}\left(\mathbf{e}_{n}^{T}\left(A^{-1} B\right)^{k-1} \mathbf{u}\right)\left(\mathbf{e}_{n}^{T}\left(A^{-1} B\right)^{2 j-k-1} \mathbf{u}\right) \\
& =\frac{2}{(2 j) !} \mathbf{e}_{n}^{T} \mathbf{u}^{2 j}+\sum_{k=1}^{2 j-1}(-1)^{k} \frac{1}{k !(2 j-k) !}\left(\mathbf{e}_{n}^{T} \mathbf{u}^{k}\right)\left(\mathbf{e}_{n}^{T} \mathbf{u}^{2 j-k}\right) \\
& =\frac{2}{(2 j) !} n^{2 j}+\sum_{k=1}^{2 j-1}(-1)^{k} \frac{1}{k !(2 j-k) !} n^{k} n^{2 j-k} \\
& =n^{2 j} \sum_{k=0}^{2 j}(-1)^{k} \frac{1}{k !(2 j-k) !}=\frac{n^{2 j}}{(2 j) !} \sum_{k=0}^{2 j}(-1)^{k}\left(\begin{array}{c}
2 j \\
k
\end{array}\right)=0 .
\end{aligned}
$$

The first non null term in the series is the one of order $p+2$ :

$$
\begin{aligned}
h^{p+2} & \left(\mathbf{y}_{0}^{T} S(J S)^{p+2} \mathbf{y}_{0}\right)\left[2\left(\mathbf{e}_{n}^{T}\left(A^{-1} B\right)^{p+1} \mathbf{u}\right)\right. \\
& \left.+\sum_{k=1}^{p+1}(-1)^{k}\left(\mathbf{e}_{n}^{T}\left(A^{-1} B\right)^{k-1} \mathbf{u}\right)\left(\mathbf{e}_{n}^{T}\left(A^{-1} B\right)^{p-k+1} \mathbf{u}\right)\right] .
\end{aligned}
$$

Since the dimension $N$ of both matrices $A$ and $B$ is proportional to $1 / h$, it is not possible to deduce so easily that such term is $O\left(h^{p+2}\right)$. Such circumstance does hold true for symmetric methods (of even order). Theorem 1 will show that the term in square brackets in the above expression is indeed $O(1)$. 
We begin with two lemmas.

Lemma 4. A BVM of order $p$ satisfies

$$
\begin{gathered}
A \mathbf{u}^{p+1}-(p+1) B \mathbf{u}^{p}=c_{p+1} \mathbf{e}+\boldsymbol{\xi}_{p+1}, \\
A \mathbf{u}^{p+2}-(p+2) B \mathbf{u}^{p+1}=d_{p+2} \mathbf{u}+c_{p+2} \mathbf{e}+\boldsymbol{\xi}_{p+2},
\end{gathered}
$$

with $d_{p+2}=(p+2) c_{p+1}$ and $\boldsymbol{\xi}_{p+1}$ and $\boldsymbol{\xi}_{p+2}$ with non null entries only in correspondence of the initial and final methods. If furthermore the basic method is symmetric, one has also $d_{p+2}=-2 c_{p+2}$.

Proof. We denote by $\boldsymbol{\alpha}$ and $\boldsymbol{\beta}$ the vectors of length $k$ that contain the coefficients $\alpha_{i}$ and $\beta_{i}$ of the main method. We consider the vector $\mathbf{w}(t)=[t-r+1, t-$ $r, \ldots, t+r]^{T}$, with $t \in \mathbb{R}$. Since the method has order $p$, it follows that

$$
\boldsymbol{\alpha}^{T} \mathbf{w}^{p}(t)-p \boldsymbol{\beta}^{T} \mathbf{w}^{p-1}(t)=0 .
$$

Integrating with respect to $t$ yields

$$
\frac{1}{p+1} \boldsymbol{\alpha}^{T} \mathbf{w}^{p+1}(t)-\boldsymbol{\beta}^{T} \mathbf{w}^{p}(t)=\tilde{c}_{p+1},
$$

from which (10) follows with $c_{p+1}=(p+1) \tilde{c}_{p+1}$. Integrating (12) once again we obtain

$$
\frac{1}{p+2} \boldsymbol{\alpha}^{T} \mathbf{w}^{p+2}(t)-\boldsymbol{\beta}^{T} \mathbf{w}^{p+1}(t)=c_{p+1}(t+\nu)+\tilde{c}_{p+2},
$$

with $\nu$ an arbitrary integer. Choosing suitably values of $t$ and $\nu$, the above expression is seen to be equivalent to the generic component of (11), with $d_{p+2}=$ $(p+2) c_{p+1}$. In particular, if the main method is symmetric with $2 r+1$ steps, one has $\boldsymbol{\alpha}=-P \boldsymbol{\alpha}$ and $\boldsymbol{\beta}=P \boldsymbol{\beta}$, with $P$ the permutation matrix having unitary elements on the secondary main diagonal. In such a case, to obtain (11) we must choose $\nu=0$. Let us set

$$
\begin{aligned}
& \mathbf{w}_{0}=\mathbf{w}(0)=[-(r+1), \ldots,-1,0, \ldots, r]^{T}, \\
& \mathbf{w}_{1}=\mathbf{w}(1)=[-r, \ldots, 0,1, \ldots, r+1]^{T} ;
\end{aligned}
$$

from (13) we have

$$
\begin{aligned}
& d_{p+2}+c_{p+2}=\boldsymbol{\alpha}^{T} \mathbf{w}_{1}^{p+2}-(p+2) \boldsymbol{\beta}^{T} \mathbf{w}_{1}^{p+1}, \\
& c_{p+2}=\boldsymbol{\alpha}^{T} \mathbf{w}_{0}^{p+2}-(p+2) \boldsymbol{\beta}^{T} \mathbf{w}_{0}^{p+1} .
\end{aligned}
$$

From the relation $\mathbf{w}_{0}^{j}=(-1)^{j} P \mathbf{w}_{1}^{j}$, that holds for any integer $j$ we obtain

$$
\begin{aligned}
c_{p+2} & =\boldsymbol{\alpha}^{T} P \mathbf{w}_{1}^{p+2}+(p+2) \boldsymbol{\beta}^{T} P \mathbf{w}_{1}^{p+1}=-\boldsymbol{\alpha}^{T} \mathbf{w}_{1}^{p+2}+(p+2) \boldsymbol{\beta}^{T} \mathbf{w}_{1}^{p+1} \\
& =-\left(d_{p+2}+c_{p+2}\right)
\end{aligned}
$$

and hence the assertion. 
Lemma 5. The extension of (8) to the indices $k=p+1$ and $k=p+2$ are respectively

$$
\left(A^{-1} B\right)^{p} \mathbf{u}=\frac{1}{(p+1) !}\left(\mathbf{u}^{p+1}+c_{12} \mathbf{u}+c_{11} \mathbf{e}+\hat{\boldsymbol{\xi}}_{1}\right)
$$

and

$$
\left(A^{-1} B\right)^{p+1} \mathbf{u}=\frac{1}{(p+2) !}\left(\mathbf{u}^{p+2}+c_{23} \mathbf{u}^{2}+c_{22} \mathbf{u}+c_{21} \mathbf{e}+\hat{\boldsymbol{\xi}}_{2}\right),
$$

where the constants $c_{i j}$ satisfy the following relations:

$$
c_{12}=-c_{p+1}, \quad c_{23}=-(p+2) c_{p+1}, \quad c_{22}=(p+2) c_{11} .
$$

Proof. Multiplying (10) on the left by the inverse of $A$ and using (d) of Lemma 1 and (a) of Lemma 3 we obtain

$$
\left(A^{-1} B\right) \mathbf{u}^{p}=\frac{1}{p+1}\left(\mathbf{u}^{p+1}-c_{p+1} \mathbf{u}+c_{11} \mathbf{e}+\hat{\boldsymbol{\xi}}_{1}\right),
$$

and considering Lemma 2 for $k=p$, we deduce

$$
\begin{aligned}
\left(A^{-1} B\right)^{p} \mathbf{u} & =\left(A^{-1} B\right)\left(A^{-1} B\right)^{p-1} \mathbf{u}=\frac{1}{p !}\left(A^{-1} B\right) \mathbf{u}^{p} \\
& =\frac{1}{(p+1) !}\left(\mathbf{u}^{p+1}-c_{p+1} \mathbf{u}+c_{11} \mathbf{e}+\hat{\boldsymbol{\xi}}_{1}\right)
\end{aligned}
$$

that coincides with (14), putting $c_{12}=-c_{p+1}$. With an analogous argument on formula (11) we derive

$$
\left(A^{-1} B\right) \mathbf{u}^{p+1}=\frac{1}{p+2}\left(\mathbf{u}^{p+2}-d_{p+2} A^{-1} \mathbf{u}-c_{p+2} \mathbf{u}-g_{1} \mathbf{e}-\hat{\boldsymbol{\xi}}_{p+2}\right),
$$

and using (14), Lemma 2 for $k=1$ and (b)-(c) of Lemma 1,

$$
\begin{aligned}
\left(A^{-1} B\right)^{p+1} \mathbf{u}= & \left(A^{-1} B\right)\left(A^{-1} B\right)^{p} \mathbf{u} \\
= & \frac{1}{(p+1) !}\left(A^{-1} B\right)\left(\mathbf{u}^{p+1}+c_{12} \mathbf{u}+c_{11} \mathbf{e}+\hat{\boldsymbol{\xi}}_{1}\right) \\
= & \frac{1}{(p+2) !}\left[\mathbf{u}^{p+2}-d_{p+2} A^{-1} \mathbf{u}-c_{p+2} \mathbf{u}-g_{1} \mathbf{e}-\hat{\boldsymbol{\xi}}_{p+2}\right. \\
& \left.-(p+2) \frac{c_{p+1}}{2} u^{2}+(p+2) c_{11}\left(\mathbf{u}-A^{-1} \mathbf{b}\right)+(p+2) A^{-1} B \hat{\boldsymbol{\xi}}_{1}\right] .
\end{aligned}
$$

Using (d), (a) and (c) of Lemma 3 for the terms $A^{-1} \mathbf{u}, A^{-1} \mathbf{b}$ and $A^{-1} B \hat{\boldsymbol{\xi}}_{1}$ respectively, we have 


$$
\begin{aligned}
\left(A^{-1} B\right)^{p+1} \mathbf{u}= & \frac{1}{(p+2) !}\left[\mathbf{u}^{p+2}-\frac{1}{2}\left(d_{p+2}+(p+2) c_{p+1}\right) \mathbf{u}^{2}\right. \\
& \left.-\left(\frac{d_{p+2}}{2}+c_{p+2}-(p+2) c_{11}\right) \mathbf{u}+c_{21} \mathbf{e}+\hat{\boldsymbol{\xi}}_{2}\right]
\end{aligned}
$$

with $c_{21}$ a suitable constant. Finally (15) follows exploiting the expressions for $d_{p+2}$ obtained in Lemma 4 .

Now we proceed with the proof of the superconvergence property.

Theorem 1. The solution of a symmetric BVM with $2 r+1$ steps and even order $p$ satisfies $\sigma_{n}=\sigma+O\left(h^{p+2}\right)$.

Proof. It is enough to prove that the term in square brackets in (9) is $\mathrm{O}(1)$. Considering once again the order conditions (8) and the extensions (14) and (15), this term is simplified as follows

$$
\begin{aligned}
& {\left[2\left(\mathbf{e}_{n}^{T}\left(A^{-1} B\right)^{p+1} \mathbf{u}\right)+\sum_{k=1}^{p+1}(-1)^{k}\left(\mathbf{e}_{n}^{T}\left(A^{-1} B\right)^{k-1} \mathbf{u}\right)\left(\mathbf{e}_{n}^{T}\left(A^{-1} B\right)^{p-k+1} \mathbf{u}\right)\right]} \\
& =\frac{2}{(p+2) !} \mathbf{e}_{n}^{T}\left(c_{23} \mathbf{u}^{2}+c_{22} \mathbf{u}+c_{21} \mathbf{e}+\hat{\boldsymbol{\xi}}_{2}\right)-\frac{2}{(p+1) !}\left(\mathbf{e}_{n}^{T} \mathbf{u}\right) \mathbf{e}_{n}^{T}\left(c_{12} \mathbf{u}+c_{11} \mathbf{e}+\hat{\boldsymbol{\xi}}_{1}\right) \\
& =\frac{2}{(p+1) !}\left[\left(\frac{c_{23}}{p+2}-c_{12}\right) n^{2}+\left(\frac{c_{22}}{p+2}-c_{11}\right) n+O(1)\right] .
\end{aligned}
$$

We arrive at the assertion considering that the coefficients of the terms in $\mathbf{u}^{2}$ and $\mathbf{u}$ vanish because of (16).

The superconvergence property allows us to modify the numerical solution in order to obtain a new one preserving the value of the Hamiltonian function. Starting from the numerical solution $\mathbf{y}_{n}$, we define $\mathbf{z}_{0}=\mathbf{y}_{0}$ and

$$
\mathbf{z}_{n}=\left(\frac{\mathbf{y}_{0}^{T} S \mathbf{y}_{0}}{\mathbf{y}_{n}^{T} S \mathbf{y}_{n}}\right)^{1 / 2} \mathbf{y}_{n}, \quad n=2, \ldots, N .
$$

Obviously now we have $\mathbf{z}_{n}^{T} S \mathbf{z}_{n}=\sigma$. The projection (17) together with formula (3) describes a new method sharing exactly the same convergence properties (order and error constants) of the original one. In fact, denoting by $\mathbf{g}_{n}(h)=$ $\mathbf{y}_{n}-\mathbf{y}\left(t_{n}\right)$ the error function at step $n\left(\mathbf{g}_{n}(h)=O\left(h^{p}\right)\right)$, from $\mathbf{y}_{n}^{T} S \mathbf{y}_{n}=\mathbf{y}_{0}^{T} S \mathbf{y}_{0}+$ $O\left(h^{p+2}\right)$ it follows that

$$
\begin{aligned}
\mathbf{z}_{n} & =\left(\frac{\sigma}{\sigma+O\left(h^{p+2}\right)}\right)^{1 / 2}\left(\mathbf{y}\left(t_{n}\right)+\mathbf{g}_{n}(h)\right) \\
& =\left(1+O\left(h^{p+2}\right)\right)\left(\mathbf{y}\left(t_{n}\right)+\mathbf{g}_{n}(h)\right)=\mathbf{y}\left(t_{n}\right)+\tilde{\mathbf{g}}_{n}(h),
\end{aligned}
$$

where $\tilde{\mathbf{g}}_{n}(h)$ and $\mathbf{g}_{n}(h)$ share the same $O\left(h^{p}\right)$ term. 


\section{A numerical test}

We use the ETR of order 4 (see formula (5)) to solve the linear pendulum problem $\ddot{x}+\omega^{2} x=0, \omega=10$ with initial condition $x(0)=1, \dot{x}(0)=-2$, in the time interval $[0,2 \pi]$. At each run we halve the stepsize $h$, starting from $h=2 \pi / 5$ (this will cause the doubling of the dimension $N$ of the system). In the columns of Table 1 we report

- the maximum errors in the numerical solutions $\mathbf{y}_{n}$ and $\mathbf{z}_{n}$ :

$$
E\left(\mathbf{y}_{n}\right)=\max _{1 \leq n \leq N}\left\|\mathbf{y}\left(t_{n}\right)-\mathbf{y}_{n}\right\|_{\infty}, \quad E\left(\mathbf{z}_{n}\right)=\max _{1 \leq n \leq N}\left\|\mathbf{y}\left(t_{n}\right)-\mathbf{z}_{n}\right\|_{\infty},
$$

where $\mathbf{y}\left(t_{n}\right)=\left[x\left(t_{n}\right), \dot{x}\left(t_{n}\right)\right]^{T}$;

- the computed order of convergence of $\mathbf{z}_{n}$;

- the maximum error in the approximation of the Hamiltonian function obtained by $\mathbf{y}_{n}$ :

$$
H\left(\mathbf{y}_{n}\right)=\max _{1 \leq n \leq N}\left|\sigma-\sigma_{n}\right| ;
$$

- the computed order of convergence of $\sigma_{n}$ towards $\sigma$.

As shown at the end of the last section due to the superconvergence (see last column in the table), $E\left(\mathbf{y}_{n}\right)$ and $E\left(\mathbf{z}_{n}\right)$ become eventually identical.

Table 1. Convergence properties of the solution of the linear pendulum problem obtained by the ETR of order 4

\begin{tabular}{rlcccc}
\hline$N$ & \multicolumn{1}{c}{$E\left(\mathbf{y}_{n}\right)$} & \multicolumn{1}{c}{$E\left(\mathbf{z}_{n}\right)$} & ord. $\mathbf{z}_{n}$ & $H\left(\mathbf{y}_{n}\right)$ & ord. $H\left(\mathbf{y}_{n}\right)$ \\
\hline 5 & $4.06103 \cdot 10^{0}$ & $5.39728 \cdot 10^{0}$ & & $1.83 \cdot 10^{1}$ & \\
10 & $9.95836 \cdot 10^{-1}$ & $1.01165 \cdot 10^{0}$ & 2.41 & $1.52 \cdot 10^{0}$ & 3.59 \\
20 & $7.19667 \cdot 10^{-2}$ & $7.19312 \cdot 10^{-2}$ & 3.81 & $3.17 \cdot 10^{-2}$ & 5.58 \\
40 & $4.72723 \cdot 10^{-3}$ & $4.72622 \cdot 10^{-3}$ & 3.93 & $5.25 \cdot 10^{-4}$ & 5.92 \\
80 & $4.16611 \cdot 10^{-4}$ & $4.16615 \cdot 10^{-4}$ & 3.50 & $8.32 \cdot 10^{-6}$ & 5.98 \\
160 & $2.62860 \cdot 10^{-5}$ & $2.62860 \cdot 10^{-5}$ & 3.99 & $1.30 \cdot 10^{-7}$ & 6.00 \\
\hline
\end{tabular}

\section{References}

1. Aceto, L., Trigiante, D.: Symmetric schemes, time reversal symmetry and conservative methods for Hamiltonian systems, J. Comput. Appl. Math. 107 (1999) 257-274

2. Amodio, P., Brugnano, L.: On the conditioning of Toeplitz band matrices, Mathematical and Computer Modelling 23 (10) (1996) 29-42

3. Brugnano, L., Trigiante, D.: Solving ODEs by Linear Multistep Initial and Boundary Value Methods, Gordon \& Breach, Amsterdam, 1998

4. Hairer, E., Lubich, Ch., Wanner, G.: Geometric Numerical Integration StructurePreserving Algorithms for Ordinary Differential Equations. Springer Series in Computational Mathematics 31, 2002

5. Sanz-Serna, J.M., Calvo, M.P.: Numerical Hamiltonian problems. Chapman and Hall, London, 1994 